Copying. This journal is registered with the Copyright Clearance Center, 27 Congress Street, Salem, Mass. 01970. Organizations in the USA who are also registered with C.C.C. may therefore copy material (beyond the limits permitted by sections 107 and 108 of the US copyright law) subject to payment to C.C.C. of the per-copy fee of $\$ 5.00$. This consent does not extend to multiple copying for promotional or commercial purposes. Code $0007-1145 / 93 \$ 5.00+.00$. Organizations authorized by the Copyright Licensing Agency may also copy material subject to the usual conditions.

ISI Tear Sheet Service, 3501 Market Street, Philadelphia, Pennsylvania 19104, USA, is authorized to supply single copies of separate articles for private use only.

For all other use, permission should be sought from Cambridge or the American Branch of Cambridge University Press.

The Proceedings of the Nutrition Society, published by the Cambridge University Press, in part record meetings of the Symposium type, at which experts in a particular field are invited by Council to make contributions on specific parts of it and at which general discussion follows these invited contributions. The contributions will be published in extenso; such summaries of the ensuing discussions as circumstances warrant may also be published. The Society also holds scientific meetings at which papers are communicated by members and others on original work carried out by them. It is proposed at present to publish summaries of the papers read at each meeting, each communication being recorded in the Society's Proceedings by means of an abstract not exceeding in length 400 words or the equivalent space in print. The Proceedings are published three times a year.

The subscription to the Proceedings is $£ 109.00$ net (US \$217.00 in USA, Canada and Mexico). Single issues are $£ 38.00$ (US $\$ 74.00$ in the USA, Canada and Mexico) each; postage extra.

\section{INDEX OF AUTHORS}

\begin{tabular}{|c|c|c|}
\hline Aboye, C. & 593 & Gabre, P. \\
\hline Adlercreutz, $\mathrm{H}$. & 525 & Gambella, G. R \\
\hline Alférez, M. J. M. & 609 & Gebru, H. \\
\hline Aliaga, I. L. & 609 & Gill, M. \\
\hline Aman, $\mathrm{P}$. & 525 & Goodall, E. A. \\
\hline Atinmo, $\mathrm{T}$. & 439,449 & \\
\hline Ayana, G. & 593 & $\begin{array}{l}\text { Haines, M. J. } \\
\text { Hallmans, G. }\end{array}$ \\
\hline Ballard, F. J. & 393 & Hansen, I. \\
\hline Barrionuevo, M. & 609 & Hautvast, J. G. A. J. \\
\hline Barry, T. N. & 647 & \\
\hline Battistini, N. & 433 & Illman, R. J. \\
\hline Bedogni, G. & 433 & Ishibashi, T. \\
\hline Beever, D. E. & 381 & \\
\hline Berger, L. L. & 471 & James, O. F. W. \\
\hline Berry, M. & 393 & Jensen, B. B. \\
\hline Beynen, A. C. & 585 & \\
\hline Bini, A. & 433 & Kennard, J. \\
\hline Blix, A. S. & 485 & Kennedy, D. G. \\
\hline Bray, S. P. & 557 & Kennedy, S. \\
\hline Brinkman, M. & 393 & Knudsen, K. E. B. \\
\hline Cammell, S. B. & 381 & Lisbona, F. \\
\hline Campos, M. S. & 609 & Longland, A. C. \\
\hline Chandler, C. & 393 & Low, A. G. \\
\hline Clark, D. & 393 & Low, A. G. \\
\hline Clark, C. & 393 & Lundin, E. \\
\hline Clarke, J. M. & 503 & \\
\hline Costa, N. M. B. & 515 & $\begin{array}{l}\text { McNabb, W. C. } \\
\text { Marsona, Y. }\end{array}$ \\
\hline Dhanoa, M. S. & 381 & Mathers, J. C. \\
\hline Dubois, S. & 407 & Milne, J. A. \\
\hline Duncan, A. J. & 631 & $\begin{array}{l}\text { Morgan, L. M. } \\
\text { Murphy, M. R. }\end{array}$ \\
\hline Egun, G. N. & 439,449 & \\
\hline Even, P. C. & 421 & $\begin{array}{l}\text { Nestel, P. } \\
\text { Neville, S. D. }\end{array}$ \\
\hline Fisseha, T. & 593 & Nicolaïdis, S. \\
\hline France, J. & 381 & $\begin{array}{l}\text { Noblet, J. } \\
\text { Nolan, S. R. }\end{array}$ \\
\hline
\end{tabular}

Nordøy, E. S.

Okamoto, K.

Pallarés, I.

Quelch, D. B.

Shavila, Y.

Shelton, I. D.

Shi, X. S.

Smit, J. G. G.

Sørmo, W.

Southgate, D. A. T.

Stenling, R.

Tadesse, A.-S.

Tanaka, M.

Tomas, F.

Topping, D. L.

Toyomizu, M.

Travis, J. S.

Tredger, J. A.

Trimble, R. P.

van der Heide, D.

van Tintelen, $\mathrm{G}$.

van Milgen, J.

Virgili, F.

Waghorn, G. C.

Walker, A. F.

Walsh, D. M.

West, C. E.

Westerlund, E.

Withers, R. T.

Wolde-Gebriel, Z.

Wright, J.

Zhang, J.-X.
485

459

609

491

647

407

585

485

379

525

593

459

393

503

459

491

491

503

585

585

471

433

647

515

621

593

525

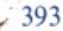

593

491

525 


\section{CONTENTS}

\section{Editorial}

Missionary zeal. D. A. T. Southgate

\section{Energy Metabolism}

Examination of energy utilization in cattle offered a forage diet at near- and submaintenance levels of feeding. S. B. Cammell, M. J. Haines, M. Gill, M. S. Dhanoa, J. France \& D. E. Beever

No major differences in energy metabolism between matched and unmatched groups of 'large-eating' and 'small-eating' men. Dallas Clark, Frank Tomas, Robert T. Withers, Sally D. Neville, Stephen R. Nolan, Menno Brinkman, Colin Chandler, Carol Clark, F. John Ballard, Michael Berry \& Paul Nestel

Metabolic utilization of dietary energy and nutrients for maintenance energy requirements in sows: basis for a net energy system. J. Noblet, X. S. Shi \& S. Dubois

Adaptive changes in energy expenditure during mild and severe feed restriction in the rat. Patrick C. Even \& S. Nicolaïdis

\section{Body Composition Measurements in vivo}

In vivo total body water assessment by total body electrical conductivity in rats suffering perturbations of water compartment equilibrium. N. Battistini, F. Virgili, G. Bedogni, G. R. Gambella \& A. Bini

\section{Protein Metabolism}

Protein requirement of young adult Nigerian females on habitual Nigerian diet at the usual level of energy intake. Gloria N. Egun \& Tola Atinmo

A metabolic nitrogen balance study for $40 \mathrm{~d}$ and evaluation of the menstrual cycle on protein requirement in young Nigerian women. Gloria N. Egun \& Tola Atinmo

\section{Metabolic Responses to Dietary Protein}

Tissue- and substrate-dependent responses to oxidative phosphorylation to dietary protein level in chicks. Masahiro Tanaka, Teru Ishibashi, Katsuyuki Okamoto \& Masaaki Toyomizu

Modelling Feed Intake, Digestion and Rumen Bacteria

An integrated, dynamic model of feed hydration and digestion, and subsequent bacterial mass accumulation in the rumen. Jaap van Milgen, Larry L. Berger \& Michael R. Murphy

\section{Digestibility of Prey by Minke Whales}

In vitro digestibility of different prey species of minke whales (Balaenoptera acutorostrata). Erling S. Nordøy, Wenche Sørmo \& Arnoldus Schytte Blix

Effects of Complex Carbohydrates on Lipid Metabolism

The effect of non-starch polysaccharide supplementation on circulating bile acids, hormone and metabolite levels following a fat meal in human subjects. L. M. Morgan, J. A. Tredger, Y. Shavila, J. S. Travis \& J. Wright

Plasma lipids and large bowel volatile fatty acids in pigs fed on white rice, brown rice and rice bran. Yustinus Marsono, Richard J. IIIman, Julie M. Clarke, Rodney P. Trimble \& David L. Topping

The effect of graded inclusion of baked beans (Phaseolus vulgaris) on plasma and liver lipids in hypercholesterolaemic pigs given a Western-type diet. Neuza M. B. Costa, Ann F. Walker \& A. G. Low 